\title{
Cronin effect at different energies: from RHIC to LHC
}

\author{
Michal Krelina ${ }^{1, a}$ and Jan Nemchik ${ }^{1,2, b}$ \\ ${ }^{1}$ Czech Technical University in Prague, FNSPE, Brehova 7, 11519 Prague, Czech Republic \\ ${ }^{2}$ Institute of Experimental Physics SAS, Watsonova 47, 04001 Kosice, Slovakia
}

\begin{abstract}
Using the QCD improved parton model we study production of hadrons with large transverse momenta $p_{T}$ in proton-proton and proton-nucleus collisions at different energies corresponding to experiments at RHIC and LHC. For investigation of large- $p_{T}$ hadrons produced on nuclear targets we include additionally the nuclear modification of parton distribution functions and the nuclear broadening calculated within the color dipole formalism. We demonstrate that complementary effect of initial state interactions causes a significant suppression at large $p_{T}$ and at forward rapidities. We provide a good description of the Cronin effect at medium-high $p_{T}$ and the nuclear suppression at large $p_{T}$ in agreement with available data from experiments at RHIC and LHC. In the LHC energy range this large- $p_{T}$ suppression expected at forward rapidities can be verified by the future measurements.
\end{abstract}

\section{Introduction}

Experimental and theoretical investigation of inclusive hadron (h) production at different transverse momenta $p_{T}$ in proton-nucleus $(p+A)$ with respect to proton-proton $(p+p)$ collisions allows to study various nuclear phenomena through the nucleus-to-proton ratio, the so called nuclear modification factor, $R_{A}\left(p_{T}\right)=\sigma_{p+A \rightarrow h+X}\left(p_{T}\right) / A \sigma_{p+p \rightarrow h+X}\left(p_{T}\right)$, where $A$ is the mass number.

The Cronin effect, observed already in 1975 [1] as the ratio $R_{A}\left(p_{T}\right)>1$ at medium-high $p_{T}$, was studied in [2] within the color dipole formalism. Predicted magnitude and the shape of this effect was verified later by the PHENIX data [3] at RHIC and recently by the ALICE experiment [4] at LHC. However, other models presented in [5] do not provide a good description of the last ALICE data [4].

Besides Cronin enhancement of particle production at medium-high $p_{T}$ the PHENIX data [3] on $\pi^{0}$ production in $d+A u$ collisions at mid rapidity $(y=0)$ indicate a suppression at large $p_{T}, R_{A}\left(p_{T}\right)<1$. Moreover, the BRAHMS and STAR data [6] at forward rapidities demonstrate even much stronger suppression. This forward region is expected to be studied also at LHC since the target Bjorken $x$ is $e^{y}$ times smaller than at $y=0$. This allows to investigate a stronger onset of coherent phenomena (shadowing, Color Glass Condensate (CGC)), which are expected to suppress particle yields.

Interpretations of large- $y$ suppression at RHIC and LHC via CGC should be done with a great care since the assumption that CGC is the dominant source of suppression leads to severe problems with understanding of a wider samples of data at smaller energies (see examples in [7]) where no coherence effects are possible. This supports a manifestation of another mechanism proposed in [7] and applied for description of various processes in $p(d)+A$ interactions [8] and in heavy ion collisions [9]. Such a

\footnotetext{
a e-mail: michal.krelina@fjfi.cvut.cz

be-mail: nemcik@saske.sk
} 
mechanism is valid at any energy and is responsible for a significant suppression of particle production at $\xi \rightarrow 1$, where $\xi=\sqrt{x_{F}^{2}+x_{T}^{2}}$ with Feynman $x_{F}$ and variable $x_{T}=2 p_{T} / \sqrt{s}$ defined at given c.m. energy $\sqrt{s}$. Dissipation of energy due to initial state interactions (ISI) [7] leads to breakdown of the QCD factorization at large $\xi$ and we rely on the factorization formula, Eq. (4), where we replace the proton parton distribution function (PDF) by the nuclear modified one, $f_{a / p}\left(x, Q^{2}\right) \Rightarrow f_{a / p}^{(A)}\left(x, Q^{2}, b\right)$, where

$$
f_{a / p}^{(A)}\left(x, Q^{2}, b\right)=C_{v} f_{a / p}\left(x, Q^{2}\right) \frac{e^{-\xi \sigma_{e f f} T_{A}(b)}-e^{-\sigma_{e f f} T_{A}(b)}}{(1-\xi)\left(1-e^{-\sigma_{e f f} T_{A}(b)}\right)}
$$

with $\sigma_{e f f}=20 \mathrm{mb}$ and with the normalization factor $C_{v}$ fixed by the Gottfried sum rule.

\section{Cross section calculations}

For calculations of the inclusive hadron production in $p+p$ and $p(d)+A$ interactions we adopt the QCD improved parton model. The corresponding invariant inclusive cross section of the process $p+p \rightarrow h+X$ is then given by the standard convolution expression based on QCD factorization [10],

$E \frac{d^{3} \sigma^{p p \rightarrow h X}}{d^{3} p}=K \sum_{a b c d} \int d^{2} k_{T a} d^{2} k_{T b} \frac{d x_{a}}{x_{R a}} \frac{d x_{b}}{x_{R b}} F_{p}^{a}\left(x_{a}, k_{T a}^{2}, Q^{2}\right) F_{p}^{b}\left(x_{b}, k_{T b}^{2}, Q^{2}\right) D_{h / c}\left(z_{c}, \mu_{F}^{2}\right) \frac{1}{\pi z_{c}} \frac{d \hat{\sigma}^{a b \rightarrow c d}}{d \hat{t}}$

where functions $F_{p}^{i}\left(x_{i}, k_{T i}^{2}, Q^{2}\right)=x_{i} f_{i / p}\left(x_{i}, Q^{2}\right) g_{p}\left(k_{T i}^{2}, Q^{2}\right), K$ is the normalization factor, $K \approx 1.0-1.5$ depending on the energy, $x_{a}, x_{b}$ are fractions of longitudinal momentum of colliding partons, $z_{c}$ is a fraction of the parton momentum carried by a produced hadron, $d \hat{\sigma} / d \hat{t}$ is the hard parton scattering cross section and radial variable is defined as $x_{R i}^{2}=x_{i}^{2}+4 k_{T i}^{2} / s$.

The distribution of the initial parton transverse momentum is described by the Gaussian form [11]

$$
g_{p}\left(k_{T}, Q^{2}\right)=\frac{1}{\pi\left\langle k_{T}^{2}\right\rangle_{N}\left(Q^{2}\right)} e^{-k_{T}^{2} /\left\langle k_{T}^{2}\right\rangle_{N}\left(Q^{2}\right)} \quad \text { with } \quad\left\langle k_{T}^{2}\right\rangle_{N}\left(Q^{2}\right)=\left\langle k_{T}^{2}\right\rangle_{0}+0.2 \alpha_{S}\left(Q^{2}\right) Q^{2},
$$

where $\left\langle k_{T}^{2}\right\rangle_{0}=1.5 \mathrm{GeV}^{2}$ for RHIC and $\left\langle k_{T}^{2}\right\rangle_{0}=0.5 \mathrm{GeV}^{2}$ for LHC energy in order to obtain the best description of hadron spectra in $p+p$ collisions as is depicted in Fig.1. For the hard parton scattering cross section we use regularization masses $\mu_{q}=0.2 \mathrm{GeV}$ and $\mu_{G}=0.8 \mathrm{GeV}$ for quark and gluon propagators, respectively [2].

For the process $p+A \rightarrow h+X$ the corresponding invariant differential cross section reads,

$$
\begin{array}{r}
E \frac{d^{3} \sigma^{p A \rightarrow h X}}{d^{3} p}=K \sum_{a b c d} \int d^{2} b T_{A}(b) \int d^{2} k_{T a} d^{2} k_{T b} \frac{d x_{a}}{x_{R a}} \frac{d x_{b}}{x_{R b}} g_{A}\left(x_{a}, k_{T a}, Q^{2}, b\right) g_{p}\left(k_{T b}, Q^{2}\right) \\
\times x_{a} f_{a / p}\left(x_{a}, Q^{2}\right) x_{b} f_{b / A}\left(b, x_{b}, Q^{2}\right) D_{h / c}\left(z_{c}, \mu_{F}^{2}\right) \frac{1}{\pi z_{c}} \frac{d \hat{\sigma}^{a b \rightarrow c d}}{d \hat{t}},
\end{array}
$$

where $T_{A}(b)$ is the nuclear thickness function. The nuclear parton distribution functions (NPDFs) $f_{b / A}\left(x_{b}, Q^{2}\right)=R_{f}^{A}\left(x_{b}, Q^{2}\right)\left[\frac{Z}{A} f_{b / p}\left(x_{b}, Q^{2}\right)+\left(1-\frac{Z}{A}\right) f_{b / n}\left(x_{b}, Q^{2}\right)\right]$ were obtained using the nuclear modification factor $R_{f}^{A}\left(x_{b}, Q^{2}\right)$ with EPS09 [12] and nDS [13] parametrizations. The nuclear modified distribution of the initial parton transverse momentum has the form

$$
g_{A}\left(x, k_{T}, Q^{2}, b\right)=\frac{1}{\pi\left\langle k_{T}^{2}\right\rangle_{A}\left(x, Q^{2}, b\right)} e^{-k_{T}^{2} /\left\langle k_{T}^{2}\right\rangle_{A}\left(x, Q^{2}, b\right)}, \quad \text { where } \quad\left\langle k_{T}^{2}\right\rangle_{A}\left(Q^{2}, b\right)=\left\langle k_{T}^{2}\right\rangle_{N}\left(Q^{2}\right)+\Delta k_{T}^{2}(x, b) .
$$

Here $\Delta k_{T}^{2}(x, b)=2 C(x) T_{A}(b)$ [14] represents nuclear broadening (NB) evaluated within the color dipole formalism. The factor $C(x)$ is related to the dipole cross section $\sigma_{\bar{q} q}$ as $C(x)=$ 



Figure 1. Single inclusive hadron spectra in $p+p$ collisions vs. data at $\sqrt{s}=200$ and $2760 \mathrm{GeV}$.

$d \sigma_{\bar{q} q}(x, r) /\left.d r^{2}\right|_{r=0}$. NB for gluons is larger due to the Casimir factor 9/4. In all calculations we take the scale $Q^{2}=\mu_{F}^{2}=p_{T}^{2} / z_{c}^{2}$. For PDFs and fragmentation functions we use MSTW2008 [15] and DSS [16] parametrization, respectively. For the dipole cross section we adopt the GBW parametrization from [17] and Impact-Parameter dependent Saturation Model (IP-Sat) from [18].

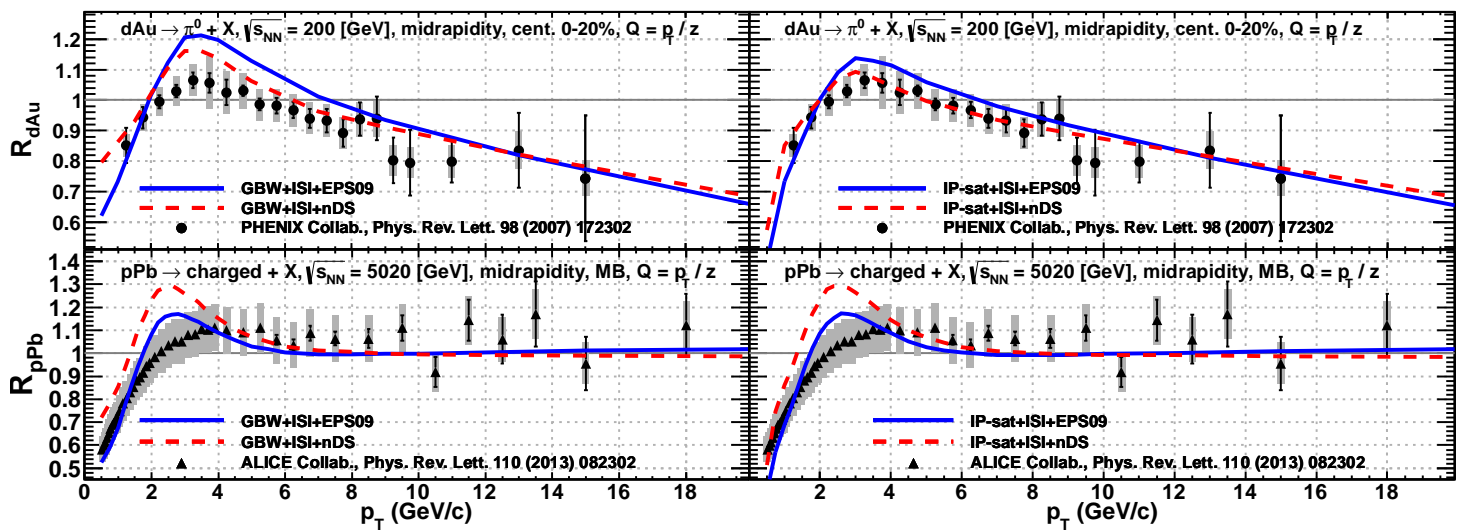

Figure 2. Prediction for the Cronin effect vs. PHENIX [3] and ALICE [4] data.

In Fig. 2 we present predictions for the Cronin effect at mid rapidity in inclusive hadron production at RHIC and LHC in a good agreement with PHENIX data [3] for central (0-20\%) $d+A u$ collisions and with data from the ALICE [4] experiment. In all calculations we include ISI effects given by Eq. (1). NPDFs with EPS09 [12] and nDS [13] parametrization are depicted by the solid and dashed lines, respectively. Nuclear broadening is calculated using GBW [17] (left boxes) and IP-Sat [18] (right boxes) parametrization of the dipole cross section, respectively. Note that ISI effects are irrelevant at LHC but cause a significant large- $p_{T}$ suppression at RHIC.

While we predict in the LHC energy range a weak onset of ISI effects at $y=0$ resulting in $R_{p+P b}\left(p_{T}\right) \rightarrow 1$ (see Fig. 2), at forward rapidities we expect a significant nuclear large- $p_{T}$ suppression as is shown in Fig. 3 for several $y=2,3$ and 4. The dotted lines represent calculations without ISI effects and NPDFs. The dashed lines include additionally ISI effects given by Eq. (1) and solid lines represent the full calculation including both ISI effects and NPDFs with EPS09 parametrization [12]. Here in all calculations we use IP-Sat parametrization [18] of the dipole cross section. 


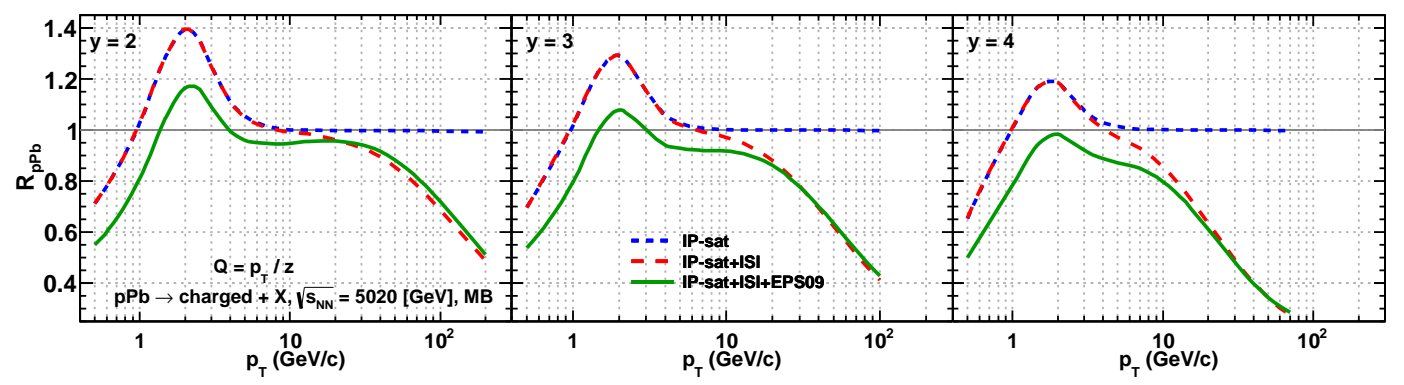

Figure 3. Nuclear modification factor $R_{p+P b}\left(p_{T}\right)$ for hadron production at $\sqrt{s}=5.02 \mathrm{TeV}$ and at $y=2,3$ and 4 .

\section{Conclusions}

We provide a good description of data on the Cronin effect at medium-high $p_{T}$ at RHIC and LHC energies adopting the QCD improved parton model. Nuclear broadening is calculated within the color dipole formalism using two different parametrizations of the dipole cross section. At large $p_{T}$ we demonstrate a strong onset of ISI effects at RHIC even at mid rapidity causing a significant suppression. In the LHC kinematic region ISI effects are irrelevant at $y=0$ but we predict a strong large- $p_{T}$ suppression at forward rapidities that can be verified by the future measurements.

\section{Acknowledgements}

This work has been supported by the grant 13-02841S of the Czech Science Foundation (GAČR), by the Grant VZ MŠMT 6840770039, by the Slovak Research and Development Agency APVV-0050-11 and by the Slovak Funding Agency 2/0092/10.

\section{References}

[1] J.W. Cronin, et al., Phys. Rev. D. 11, 3105 (1975).

[2] B.Z. Kopeliovich, et al., Phys. Rev. Lett. 88, 232303 (2002).

[3] S.S. Adler, et al. (PHENIX Collaboration), Phys. Rev. Lett. 98, 172302 (2007).

[4] B. Abelev, et al. (ALICE Collaboration), Phys. Rev. Lett. 110, 082302 (2013).

[5] J. Albacete, N. Armesto, et al., Int. J. Mod. Phys. E22, 1330007 (2013)

[6] I. Arsene, et al. (BRAHMS Collaboration), Phys. Rev. Lett. 93, 242303 (2004); J. Adams, et al. (STAR Collaboration), Phys. Rev. Lett. 97, 152302 (2006).

[7] B. Z. Kopeliovich et al., Phys. Rev. C72, 054606 (2005); B. Z. Kopeliovich and J. Nemchik, J. Phys. G38, 043101 (2011).

[8] J. Nemchik, et al., Phys. Rev. C78, 025213 (2008); Nucl. Phys. A830, 611c (2009).

[9] B.Z. Kopeliovich and J. Nemchik, Phys. Rev. C86, 054904 (2012).

[10] R. P. Feynman, R. D. Field and G. C. Fox, Phys. Rev. D18, 3320 (1978).

[11] X. N. Wang, Phys. Rev. C61, 064910 (2000).

[12] K. J. Eskola, H. Paukkunen and C. .A. Salgado, JHEP 0904, 065 (2009).

[13] D. de Florian and R. Sassot, Phys. Rev. D69, 074028 (2004).

[14] M. B. Johnson, B. Z. Kopeliovich and A. V. Tarasov, Phys. Rev. C63, 035203 (2001).

[15] A. D. Martin, W. J. Stirling, R. S. Thorne and G. Watt, Eur. Phys. J. C63, 189-285 (2009).

[16] D. de Florian, R. Sassot and M. Stratmann, Phys. Rev. D75, 114010 (2007).

[17] K. Golec-Biernat and M. Wüsthoff, Phys. Rev. D59, 014017 (1998).

[18] A. H. Rezaeian, at al., Phys. Rev. D87, 034002 (2013). 